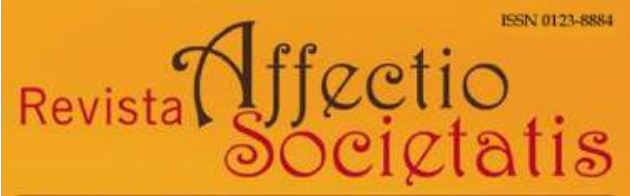

Departamento de Psicoanálisis | Universidad de Antioquia

Revista Affectio Societatis

Departamento de Psicoanálisis

Universidad de Antioquia

revistaaffectiosocietatis@udea.edu.co

ISSN (versión electrónica): 0123-8884

Colombia

Idamari Santiago

La presencia-ausencia del objeto en la anorexia y en la bipolaridad

Revista Affectio Societatis, Vol. 17, N. ${ }^{\circ} 32$, enero-junio de 2020 Art. \# 8 (pp.178-190)

Departamento de Psicoanálisis, Universidad de Antioquia

Medellín, Colombia 


\section{LA PRESENCIA-AUSENCIA DEL OBJETO EN LA ANOREXIA Y EN LA BIPOLARIDAD ${ }^{1}$}

Idamari Santiago Castro ${ }^{2}$ Universidad de Puerto Rico, Recinto de Río Piedras

idamari.santiago@upr.edu ORCID: 0000-0001-7977-8015 DOI: 10.17533/udea.affs.v17n32a08

\section{Resumen}

En este artículo reflexivo se pretende pensar e interrogar a la anorexia y la bipolaridad, no como categorías nosológicas, sino respecto de la economía afectiva del sujeto a partir de la relación de objeto. Se propone considerar a la anorexia en relación con la hiperausencia del objeto: el sujeto come nada; mientras que a la bipo- laridad como un circuito en que el sujeto pasa de la hiperpresencia a la hiperausencia del objeto: el sujeto se encuentra atrapado en el circuito manía- melancolía.

Palabras clave: Anorexia, bipolaridad, relación de objeto, economía afectiva, dialéctica presencia-ausencia.

\section{THE PRESENCE-ABSENCE OF THE OBJECT IN ANOREXIA AND BIPOLARITY}

\section{Abstract}

This reflective paper seeks to think egories, but concerning the affective about and question anorexia and economy of the subject based on the bipolarity, not as nosological cat- object relation. It is proposed to con-

1 El presente artículo forma parte del trabajo de tesis doctoral titulado: "Dialéctica presencia-ausencia: Reflexiones sobre la relación de objeto y los afectos en la clínica del sujeto, realizada en la Universidad de Puerto Rico, Recinto de Río Piedras en el año 2016.

2 Doctora en Filosofía con especialidad en Psicología Clínica, Universidad de Puerto Rico, Recinto de Río Piedras. 
sider anorexia in relation to the hyperabsence of the object: the subject eats nothing; while bipolarity as a circuit in which the subject moves from the hyper-presence to the hyper-absence of the object: the subject is trapped in the mania-melancholia circuit.

Keywords: Anorexia, bipolarity, object relation, affective economy, presence-absence dialectic.

\section{LA PRÉSENCE-ABSENCE DE L'OBJET DANS L'ANOREXIE ET LA BIPOLARITÉ}

\section{Résumé}

Cet article a pour but de penser et d'interroger l'anorexie et la bipolarité non pas en tant que catégories nosologiques, mais en ce qui concerne l'économie affective du sujet à partir de la relation d'objet. L'approche proposée considère, d'une part, l'anorexie par rapport à l'hyper-absence de l'objet : le sujet mange du rien; d'une autre part, la bipolarité en tant que circuit où le sujet passe de l'hyper-présence à l'hyper-absence de l'objet : le sujet se trouve coincé dans le circuit manie- mélancolie.

Mots-clés : anorexie, bipolarité, relation d'objet, économie affective, dialectique présence-absence.

Recibido: 20/05/2019 • Aprobado: 10/09/2019 
El énfasis de este artículo girará en torno a la importancia de tener en cuenta la dinámica dialéctica de la presencia-ausencia para pensar la relación del sujeto con el objeto. Se entiende que es de suma importancia poder enlazar la relación de objeto y los afectos con las categorías sintomáticas de la anorexia y la bipolaridad para también brindar una perspectiva crítica sobre estas desde el psicoanálisis. Se propone pensar a la anorexia y la bipolaridad no como patologías psiquiátricas, sino abordarlas a partir de lo que el psicoanálisis ha perfilado respecto a las mismas, particularmente, en su relación con la economía afectiva del sujeto. Se trabajan ambas categorías como modos de seren-el-mundo, o dicho de otra manera, se piensan como síntomas que dan cuenta de distintas modalidades de goce relacionadas a la hiperausencia (anorexia) y al circuito de hiperpresencia-hiperausencia del objeto (bipolaridad). Se utiliza el elemento compositivo hiper al hablar de la dialéctica presencia-ausencia en relación con estos modos de goce, ya que el mismo está relacionado con lo excesivo. En la anorexia y en la bipolaridad encontramos una relación del sujeto con el objeto que está fuera de los límites amparadores del significante.

No se pretende abarcar la amplitud de las reflexiones y debates que hay en torno a estas categorías nosológicas en el psicoanálisis, sino más bien plantear algunas coordenadas teóricas que nos permitan pensar el tema de interés en esta reflexión. Por lo tanto, buscaremos señalar los aspectos cruciales que nos ayudan a pensar respecto al supuesto entramado dialéctico y afectivo que está en juego en dichas categorías. Además, es importante adelantar en este momento que estas modalidades de goce pueden manifestarse en las diversas estructuras subjetivas; es decir, no son exclusivas de una estructura en particular, como tampoco son exclusivas del hombre o de la mujer, sino que suelen manifestarse en ambos casos.

La anorexia (la hiperausencia del objeto) la pensamos como una modalidad de goce en la cual el objeto está hiperpresente como hiperausencia bajo la forma simbólica de la 'nada'. Ahora bien, anorexia ${ }^{3}$ es una palabra proveniente del griego cuyo significado en el mundo

3 La palabra anorexia proviene del griego orexis, que significa deseo. 
antiguo implica la pérdida o falta de apetito, pero su sentido en la actualidad es distinto. La anorexia de nuestros tiempos se caracteriza por el rechazo al alimento, es decir, el rechazo al objeto oral (López, 1999). Además, con gran frecuencia suele estar acompañada de la distorsión de la imagen corp-oral y del miedo excesivo a engordar. Las consecuencias más reiteradas son el adelgazamiento extremo y, en la mujer, también se añade la amenorrea (ausencia de flujo menstrual). Aquí podemos observar la complejidad del fenómeno de la anorexia de nuestra época; la anorexia es una modalidad de goce cuyo síntoma es el rechazo al alimento. No olvidemos que los síntomas también dan cuenta de la relación que sostiene el sujeto con el Otro del discurso imperante del momento; por lo tanto, el rechazo al alimento en la Edad Media, por ejemplo, tiene distintas implicaciones respecto al rechazo del alimento en los tiempos actuales caracterizados por el hiperconsumo.

Decimos que la anorexia es un fenómeno transclínico, pues un sujeto puede presentar el síntoma anoréxico como resultado de una idea paranoica delirante relacionada al envenenamiento de la comida (Cosenza, 2014); el sujeto se rehúsa a comer porque tiene la certeza de que su comida ha sido envenenada por un Otro persecutorio. En la melancolía psicótica también podríamos observar el síntoma anoréxico, ya que el sujeto puede rechazar la comida como un modo más de negativa a todo lo relacionado con el mundo exterior y al deseo como deseo del Otro. A pesar de este carácter transclínico de la anorexia que también podría constatarse en algunas estructuras perversas por la relación que pudiera tener respecto a la desmentida de la falta en el Otro o respecto a, por ejemplo, un posicionamiento exhibicionista que procure el goce del Otro-, se suele observar a menudo en las neurosis por su relación con la demanda al Otro. La anorexia, como síntoma histérico podría estar más vinculada a la demanda de amor y a la pregunta sobre cómo hacer desear al Otro, la cual lleva al sujeto a responder a su deseo como deseo insatisfecho. Mientras que, por otro lado, la anorexia como síntoma obsesivo podría estar más vinculada a los rituales de ejercicios y de control del rechazo de la comida, así como a un intento de evitar todo lo relacionado con lo sexual y, de este modo, elevar el cuerpo que goza a un cuerpo idealizado que lleva al sujeto a responder a su deseo como deseo imposible. 
Por efecto del lenguaje somos falta en ser, somos ser de vacío, somos sujetos marcados por la ausencia. Es por esto, por lo que el humano no solo busca la satisfacción de sus necesidades fisiológicas, sino que también busca satisfacerse más allá, busca un plus de satisfacción, un plus de goce. En el Seminario La relación de objeto, Lacan (1994/1956-1957) nos plantea que el síntoma de la anorexia mental "no es un no comer, sino un no comer nada. Insisto -eso significa comer nada" (p. 187); nos dice que 'nada' (objeto oral vaciado) es algo que existe en el plano simbólico (pp. 187-191). Por ejemplo, esto lo constatamos cuando el bebé, a pesar de haberse alimentado de la leche materna, sigue succionando esa 'nada' más allá de la satisfacción de la necesidad. En términos lacanianos, el bebé "saborea la ausencia" (p. 187). ¿Por qué? Porque depende de la madre para satisfacer su necesidad fisiológica, pero por la 'nada' que consume hace que la madre dependa de él. A esto Lacan lo denomina la "dialéctica simbólica de la actividad oral" (p. 187). Sin embargo, la madre se hace omnipotente al negarse a satisfacer ese más allá de la necesidad. Para aclararlo mejor, el Otro materno se satisface en satisfacer la necesidad fisiológica del bebé, pero es incapaz de dar el don de amor, esa 'nada', ese don de lo que no se tiene que vale como signo de amor (Lacan, 1994/1956-1957). Para que el objeto oral se manifieste como don de amor tiene que poder faltar; en el caso del sujeto anoréxico, el objeto oral parece no faltar nunca y, como consecuencia, este se identifica con el objeto pulsional-oral-nada, por lo tanto, queda fijado al mismo. Dicho de otro modo, la anorexia hace visible su falta en ser a través del goce de su cuerpo que está ligado al objeto $a$.

En este sentido, el síntoma anoréxico está estrechamente relacionado con la dialéctica de la demanda de la satisfacción de la necesidad (demanda transitiva al Otro que tiene), con la demanda de amor (demanda intransitiva al Otro que no tiene), y con el deseo, que está más acá de la necesidad y más allá de la demanda. Cuando el objeto de la necesidad pasa a ser objeto de don es que surge el deseo. ¿Qué quiere el sujeto anoréxico? De alguna manera, y aunque pudiera parecer algo paradójico, quiere comer 'nada', quiere experimentar la ausencia. Detrás del síntoma anoréxico hay una intensa demanda de amor dirigida al Otro, una demanda donde no median las palabras, pero a través de su síntoma, el sujeto anoréxico quiere dejar saber que 
su deseo no se reduce al plano de la necesidad (Lacan, 2003/1958). Es interesante puntualizar que dicha demanda de amor se manifiesta en la oralidad. ¿A qué se debe esto? Se debe a que, en las primeras interacciones del pequeño sujeto con el Otro, el objeto oral suele ser el primer objeto que el Otro ofrece, no sólo para satisfacer la necesidad, sino como signo de amor. En el caso del sujeto anoréxico, al no recibir el don de amor por parte del Otro (esa 'nada' que demanda), entonces es capaz de producir el plus de goce al satisfacerse en esa acción de 'comer nada'. De este modo, el sujeto busca preservar su deseo para no verse reducido a un organismo que sólo se satisface fisiológicamente, pues, no debemos olvidar que el deseo siempre queda insatisfecho porque es eso que aparece siempre como imposible de colmar. La relación que el sujeto anoréxico sostiene con el Otro es una relación que indica que el Otro confunde la necesidad con el amor, es decir, que confunde la satisfacción de la necesidad con la satisfacción de la demanda de amor (Lacan, 2003/1958).

En el amor hay reconocimiento del sujeto deseante, del sujeto en falta, pero esto es lo que el Otro no reconoce (según percibe el anoréxico), y al no reconocerlo es incapaz de dar el don de amor. Por lo tanto, el sujeto anoréxico con su síntoma de rechazo al alimento y por su hastío (afecto predominante) busca sostenerse como sujeto en falta, como sujeto deseante, para compensar la frustración de la demanda de amor. Aquí podemos observar el complejo entramado afectivo que está en juego en la anorexia. De algún modo, el afecto de la frustración de la demanda de amor puede llevar a un sujeto a la anorexia, al hastío, al desgano. Por otro lado, el estrago materno, en tanto obstáculo para la separación, también puede llevar a un sujeto a la anorexia para protegerse de la angustia que le produce el goce de la madre devoradora. Cuando el Otro materno solo se ocupa de satisfacer la necesidad, no está operando como madre simbólica, en tanto no entra en la imprescindible alternancia dialéctica de la presencia-ausencia. El Otro se tendría que ausentar para dar lugar al deseo del sujeto y, de este modo, cuando ofrece el objeto oral, el sujeto lo recibe como un don. Por lo tanto, la hiperpresencia del Otro puede, asimismo, llevar a un sujeto a la anorexia. Ahora bien, el deseo en la anorexia es un pseudo-deseo, porque en la anorexia hay ausencia de sujeto; por esto el anoréxico lucha incansablemente, ya que su fin 
es garantizarse como sujeto en falta. Su deseo es deseo de 'nada', un deseo que lo puede precipitar a la nada más radical, a la muerte. No olvidemos que siempre hay algo de lo mortífero en la satisfacción pulsional; tampoco podemos perder de vista que el deseo no es deseo de ningún objeto en particular, y es por esto que no puede ser colmado jamás.

El Otro, por decirlo de alguna manera, ignora la demanda de amor abarrotando de objetos al sujeto con el fin imposible de obturar la falta estructural. El sujeto anoréxico combate la omnipotencia del Otro cuando dice que 'no'; pero no dice 'no' a la acción de comer, sino que su negatividad se da en el plano del objeto que se manifiesta en lo simbólico como 'nada' (Lacan, 1994/1956-1957). En este sentido, el sujeto anoréxico se encuentra en una lucha con el Otro, y al comer 'nada' hace al Otro dependiente, lo hace su esclavo, por lo que se constituye en su amo. Aquí vemos cómo se invierte la relación de dependencia, pues ahora el Otro está a merced de la "omnipotencia" del sujeto; es decir, cuando el sujeto anoréxico rechaza el alimento se está negando a satisfacer la demanda del Otro. También la anorexia podría pensarse como un llamado a la función paterna para que hiera la omnipotencia materna y le permita al sujeto salir del deseo materno loco y sin límites. En este sentido, el síntoma anoréxico, a diferencia del síntoma fóbico, puede pensarse como otro modo de llamar al padre en su función simbólica de corte y límite. El rechazo al alimento es el síntoma que le permite al sujeto 'separarse' simbólicamente del Otro cuando falla la función paterna. A partir de esta lógica, el sujeto anoréxico ofrece su 'nada' encarnada, su apetito de muerte, pues con esto le dice al Otro, "puedes perderme", y de este modo abre un agujero, una falta en el Otro omnipotente (Lacan, 1987/1964).

Podemos decir que en la anorexia hay una lucha con el Otro de la demanda, pero también hay una lucha con el Otro del espejo que le devuelve una imagen indeseada. El Otro del espejo le devuelve una imagen llena, una imagen que refleja un cuerpo obeso, un cuerpo en exceso a causa del abarrotamiento del objeto oral, y, en este sentido, se constituye en una imagen que perturba al sujeto, que angustia al sujeto. ¿Por qué lo angustia? Porque en esa imagen del cuerpo deformado aparece la mirada en tanto objeto (Lacan, 1987/1964); la mi- 
rada mira al sujeto, pero le muestra su ser de objeto, por lo tanto, el anoréxico desprecia la imagen corporal reflejada en el espejo, ya que aniquila su ser marcado por la falta, que es eso que quiere sostener comiendo 'nada'. Y, como come 'nada', no engorda 'nada'. ¿Qué quiere decir esto? Quiere decir que su cuerpo no se reduce al organismo, que su cuerpo es un cuerpo que goza, que su cuerpo es un cuerpo real, pero también que su cuerpo es un cuerpo imaginario y un cuerpo que ha sido afectado por el lenguaje.

Hay, según Cosenza (2014), un goce no sólo en comer 'nada', sino en verse en el espejo y en constatar así su ser de objeto. En la imagen en el espejo, el sujeto anoréxico ve el cumplimiento de la completud imaginaria del Otro; ve una imagen llena a la que parece no faltarle nada. Por esta razón, cuando se mira en el espejo se siente sometido al Otro; ve que el Otro puede ofrecer el objeto oral que colma la falta en ser, y esto angustia al sujeto, pero sabemos que el Otro no puede ofrecer dicho objeto porque también le falta. Ahora bien, si por el contrario el Otro diera signos de su falta, el sujeto podría interpretarlo como signo de amor, pero esto es, justamente, lo que el Otro omnipotente no puede dar. En la anorexia, por lo tanto, no solo está en juego la hiperpresencia del objeto oral (simbólico) en tanto 'nada' (objeto invisible), sino la hiperpresencia del objeto $a$, de la mirada (real). En la anorexia hay dificultad para la cesión del objeto 'nada' porque dicho objeto protege al sujeto anoréxico del goce intrusivo del Otro.

Muchos sujetos que padecen la anorexia de nuestros tiempos pueden estar aferrados al ideal de la belleza de la imagen, a ese ideal de la delgadez promovido por el discurso imperante de la época que exacerba el valor de lo imaginario y rechaza el valor del sujeto deseante. El discurso capitalista de nuestros tiempos rechaza la castración y da la apariencia del 'todo' y del 'no falta nada'; es un discurso que rechaza el don simbólico del amor. Es por esta razón que en la clínica psicoanalítica el tratamiento de la anorexia neurótica, por ejemplo, no está dirigido a atender la necesidad fisiológica, ni a sugerir ni a obligar al sujeto a que coma, sino que está dirigido a que el sujeto pueda constituirse realmente en sujeto en falta para que, de este modo, pueda preservar su deseo sin tener que alimentar, hasta el extremo más mortífero, a la pulsión oral con esa 'nada' que lo transparenta y aniquila silenciosamente. 
Se trata de que el analista pueda encontrar pistas importantes en la novela familiar del anoréxico, examinar esos significantes que marcan su cuerpo y escuchar de qué se trata su singular demanda de amor. Si hay algo crucial en la anorexia es el aplastamiento del objeto de amor por el objeto de la necesidad (Lacan, 2003/1958).

La bipolaridad (el circuito de la hiperpresencia-hiperausencia del objeto) se trabaja en este escrito como la alternancia entre la melancolía y la manía. La melancolía es, para Freud (1986/1927), un afecto caracterizado por "la sofocación cruel del superyó" (p.161), mientras que la manía es el afecto de la emancipación del 'yo' de la presión del superyó. La alternancia entre melancolía y manía, entre sofocación-superyoica y emancipación-yoica, son migraciones de investidura que dan cuenta de una serie de fenómenos de la vida anímica normal (Freud, 1986/1927). Lo central en la manía-depresión es el posicionamiento del 'yo', y el 'yo' se caracteriza por ser sede de conflictos afectivos. Por lo tanto, la pregunta importante a plantearnos es la siguiente: ¿cuándo esta alternancia se vuelve patológica? También sería importante preguntarnos cuándo esta alternancia pasa a considerarse una locura maniacodepresiva y cómo distinguirla de una psicosis maniacodepresiva.

A partir del psicoanálisis freudiano sabemos que en la melancolía hay un 'yo' empobrecido. Es decir, hay un 'yo' profundamente entristecido, transformado en deyección y sobre el cual ha caído la sombra del objeto. El sujeto sufre de un exceso de culpa y auto reproches que aniquilan su ser de deseo. Las recriminaciones que hace el melancólico al objeto amado se desplazan al 'yo' destrozando, así, el lazo social con el Otro (Freud, 1984/1915). Ahora bien, la manía es un estado afectivo caracterizado por un exceso de excitación, por la desinhibición y por la fuga de ideas. La fuga de ideas, que es la característica esencial en la manía, es una sucesión sin límite de $S_{1}\left(S_{1}, S_{1}, S_{1} \ldots\right)$, en lugar de la articulación significante $S_{1}-S_{2}$ que produce efectos de significación y que representa al sujeto. No hay condensación del goce porque es "la no función del objeto $a^{\prime \prime}$ (Lacan, 2006/1962-1963, p. 363).

Soler (2008) nos enseña que en la fuga de ideas del maníaco no hay punto de basta o punto de capitón, pues hay un "desenfreno de 
la palabra". Hay una exaltación del humor, alegría excesiva, euforia, ausencia de límites y descontrol. El 'yo' en la manía sale de su empobrecimiento para transformarse, periódicamente, en un 'yo' empoderado. Quizás éste sea el mayor enigma del fenómeno de la bipolaridad, pues suele ser una incógnita la trasformación de la melancolía en manía, y viceversa.

En la manía, precisemos enseguida que es la no función de $a$ lo que está en juego, y no simplemente su desconocimiento. En ella el sujeto no tiene el lastre de ningún $a$, lo cual lo entrega, sin posibilidad alguna a veces de liberarse, a la pura metonimia, infinita y lúdica, de la cadena significante. (Lacan, 2006/1962-1963, p. 363).

En el estado maníaco el 'yo' se confunde con el Ideal. Por esta razón, la manía puede pensarse como una locura de omnipotencia y omnipresencia en la que, según nos dice Soler (2008), "el sujeto se consagra a la metonimia de objetos" (p. 62); por esto no es extraño observar que en estados de manía el sujeto manifieste su excitación en una adquisición excesiva de objetos a través, por ejemplo, de compras compulsivas que prontamente lo dejan endeudado y vaciado, con todas las resonancias que estos dos significantes puedan tener. En este sentido, decimos que hay una hiperpresencia del objeto; el 'yo' siente que 'todo' puede lograrse, que puede hacerlo 'todo', que puede entrometerse en 'todo'; hay un exceso de sin-límites que se sostiene de un profundo engaño de hiperpotencia.

En la melancolía el sujeto está apasionado por la 'nada', pero en la manía el sujeto está apasionado por el 'todo'. En la melancolía hay un exceso de pesimismo, pero en la manía hay un exceso de optimismo. En la melancolía el 'yo' se empobrece, pero en la manía el 'yo' se engrandece. En la melancolía el 'yo' se coloca en posición de esclavo, pero en la manía el 'yo' se coloca en posición de amo. En la melancolía el 'yo' está en posición de sumisión, pero en la manía el 'yo' está en posición de dominio. En la melancolía el 'yo' está afectado por la pérdida, pero en la manía el 'yo' está afectado por la ganancia (aunque engañosa). En la melancolía el 'yo' no sabe qué perdió en su pérdida, en la manía el 'yo' no sabe qué ganó en su triunfo. En la melancolía hay hemorragia libidinal, en la manía hay ligazón de investiduras li- 
bres. En la melancolía el 'yo' está identificado con el objeto $a$, pero en la manía el 'yo' está identificado con el objeto fálico (con el puro goce). Por lo tanto, no es incorrecto decir que la manía es la otra cara de la melancolía. De hecho, para Freud (1984/1923) la manía es una especie de defensa contra la melancolía. Sin embargo, en ambos estados el sujeto sufre a causa de un narcisismo extremo y del exceso mortífero de la pulsión, ya sea por un empobrecimiento mortal del 'yo' (melancolía) o por una excitación mortal del 'yo' (manía). En ambos extremos hay un no saber, una profunda ignorancia del deseo.

La alternancia manía-melancolía es algo que constatamos a menudo en los dilemas afectivos de los neuróticos, pues cuando al 'yo' se le dificulta inscribir algo como pérdida, es decir, cuando se le dificulta el trabajo de duelo, suele vacilar en el circuito y alternancia de la manía y la melancolía, en el circuito-afectivo-circular-repetitivo de la hiperpresencia-hiperausencia porque no ha habido una apropiada tramitación. Una vez más el 'yo' es sede de los conflictos afectivos. Por lo tanto, cuando hablamos de psicosis maniacodepresiva (término psiquiátrico) es porque en esos casos específicos hay rechazo del inconsciente, hay rechazo del Otro, hay forclusión del Nombre del Padre (NDP), no hay responsabilidad subjetiva. ¿Qué implica esto en términos más sencillos? Pues que el sujeto "no está normado por el padre" (Acciardi, 2013, s.p.). De este modo, en la psicosis maniacodepresiva, a diferencia de la alternancia afectiva de manía-depresión que podemos observar en las neurosis, hay un retorno mortífero de lo forcluido. Precisamente, como los manuales diagnósticos de la psiquiatría contemporánea no toman en cuenta las estructuras subjetivas, el trastorno de bipolaridad suele ser una categoría nosológica sumamente confusa y poco rigurosa. En las clínicas psiquiátricas no es extraño toparse con sujetos neuróticos, así como con sujetos psicóticos, diagnosticados ambos con el mismo trastorno afectivo bipolar y siendo atendidos de modo indiferenciado, lo cual, sabemos, es sumamente perjudicial.

En la anorexia el sujeto come el objeto $a$ (objeto 'nada'), en la melancolía el sujeto no deja de no perder el objeto $a$ (objeto de 'amor'), y en la manía el sujeto no permite la función del objeto $a$ (objeto 'condensador del goce'). Cada una de estas categorías nosológicas, que desde el psicoanálisis las pensamos como síntomas y modalidades de 
goce particulares que pueden verse en cualquiera de las estructuras subjetivas, nos permite entender el entramado de la dialéctica presencia-ausencia respecto a la relación de objeto y los afectos. Y si hay algo en común en estas tres categorías sintomáticas es, en palabras de Gómez (2016, s.p.), que "hay un impasse con la dialéctica significante". También lo que estas modalidades de goce tienen en común es el exceso de la pulsión de muerte. En la anorexia y en la bipolaridad (manía-melancolía), el sujeto sostiene una intensa relación con la muerte, con el Amo absoluto hegeliano. En cada una el sujeto está en realidad en posición de esclavo, y sin ninguna intención de asumir la responsabilidad humana de hacer reconocer su deseo. $Y$ en ese no asumir la responsabilidad del sujeto deseante hay una falta, pero se trata, en este caso, de una falta ética.

\section{Referencias bibliográficas}

Acciardi, M. (29 de diciembre de 2013). ¿Bipolaridad? ¿Qué bipolaridad? Recuperado de http:/ / www.telam.com.ar/notas/201312/46433-bipolaridad-que-bipolaridad.html.

Cosenza, D. (25 de enero de 2014). La anoréxica tiene una relación con el espejo que está al límite de la persecución [Entrevista en un blog]. Recuperado de https://www.lacasadelaparaula.com/es/domenico-cosenzapsicoanalista-la-anorexica-tiene-una-relacion-con-el-espejo-que-esta-allimite-de-la-persecucion-2/.

Freud, S. (1984/1915). Duelo y melancolía. En Obras Completas (Vol. 14, pp. 245-256). Buenos Aires, Argentina: Amorrortu Editores.

Freud, S. (1984/1923). El yo y el ello. En Obras Completas (Vol. 19 pp. 21-30). Buenos Aires, Argentina: Amorrortu Editores.

Freud, S. (1986/1927). El humor. En Obras Completas (Vol. 21 pp. 153-155). Buenos Aires, Argentina: Amorrortu Editores.

Gómez, M. A. (Marzo, 2016). Comunicación personal.

Lacan, J. (1987/1964). El seminario. Libro 11, Los cuatro conceptos fundamentales del psicoanálisis. Buenos Aires, Argentina: Paidós.

Lacan, J. (1994/1956-1957). El seminario. Libro 4. La relación de objeto. Buenos Aires, Argentina: Paidós.

Lacan, J. (2003/1958). La dirección de la cura y los principios de su poder. En Escritos 2 (pp. 565-626). Buenos Aires, Argentina: Siglo Veintiuno Editores. 
Lacan, J. (2003/1979). Subversión del sujeto y dialéctica del deseo en el inconsciente freudiano. En Escritos 2 (pp. 773-807). Buenos Aires, Argentina: Siglo Veintiuno Editores.

Lacan, J. (2006/1962-1963). El seminario. Libro 10, La angustia. Buenos Aires, Argentina: Paidós.

López, L. S. (1999). Anorexia: Comer nada. Una perspectiva psicoanalítica. Revista Asociación Española de Neuropsiquiatría, XIX, 72, 599-608.

Soler, C. (2008). Estudios sobre la psicosis. Buenos Aires, Argentina: Manantial. 